nach Einschätzung von zwei Dritteln der Heilberufler das Arzt-Patienten-Verhältnis in Zukunft stark prägen.

„Das Bild ist relativ homogen bei den verschiedenen Heilberufen“, berichtet Projektleiter Daniel Zehnich, stellvertretender Bereichsleiter Gesundheitsmärkte und -politik bei der apoBank. Es zeigen sich aber Schwerpunkte. So richten die Ärzte ein besonderes Augenmerk auf die Entwicklung bei den Assistenzsystemen. „Hier erwarten sie vergleichsweise starke Auswirkungen im Hinblick auf ihren beruflichen Alltag, die Arzt-
Patienten-Beziehung und die Strukturen der Gesundheitsversorgung sowie einen hohen Investitionsbedarf “, sagte Zehnich. Nur 9\% rechnen allerdings mit einem hohen wirtschaftlichen Gewinn durch die Investitionen in Assistenzsysteme.

Die befürchtete Diskrepanz zwischen Kosten und Ertrag der Digitalisierung eint alle Heilberufler. Die Umfrage zeigt, dass Anreize für ein größeres Engagement bei der Digitalisierung fehlen. Hausund Fachärzte wissen, dass die Digitalisierung keine Zukunftsmusik ist. Ein
Drittel von ihnen geht davon aus, dass sie sich innerhalb der nächsten vier Jahre durchsetzen wird. Bei der Einschätzung der Folgen marschieren Standesorganisationen und ihre Mitglieder offensichtlich nicht im Gleichschritt. „Die Organisationen schätzen die Veränderungswucht geringer ein als die Heilberufler", sagt Zehnich. Insgesamt wird deutlich, dass die Heilberufler dem Thema Digitalisierung gegenüber eine hohe Sensibilität, aber keine genaue Vorstellung von den Auswirkungen im Detail haben.

Ilse Schlingensiepen

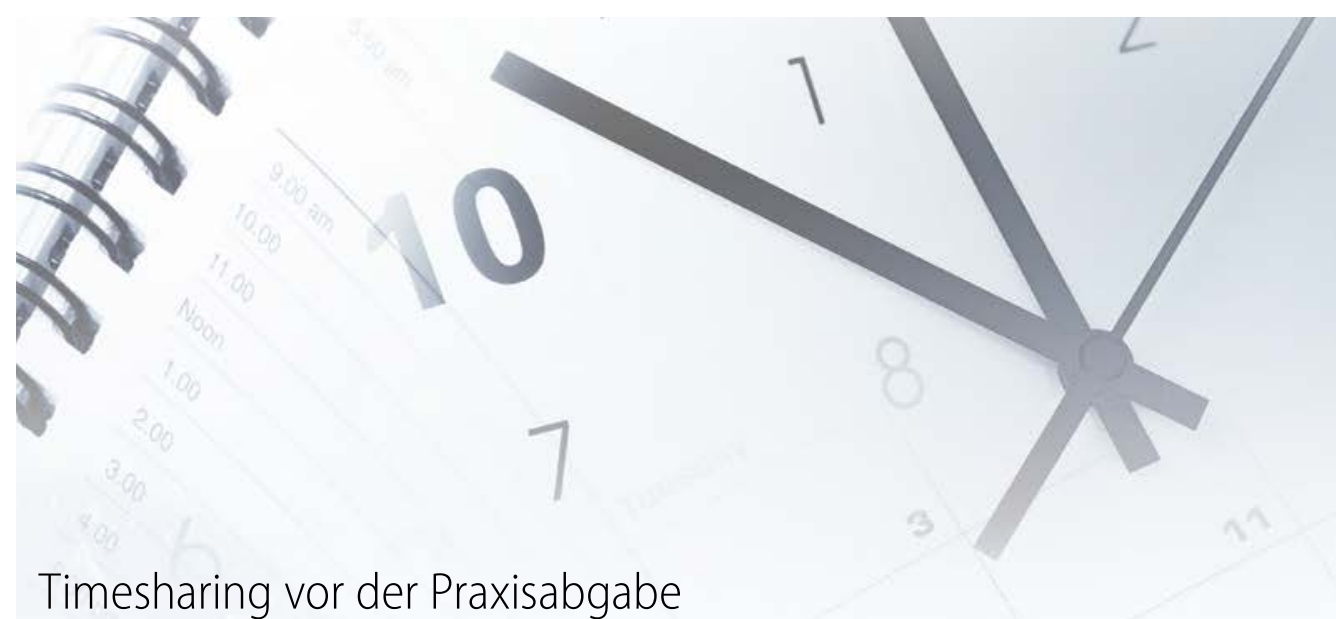

\title{
Beim Vertrag auf Nummer sicher gehen
}

\section{Das Modell des Jobsharings bis zur endgültigen Praxisübergabe bietet Vorteile für beide Seiten. Es müssen jedoch Vorgaben zur Arbeitszeit beachtet werden, die außerdem im Gesellschaftsver- trag zu regeln sind.}

Die Kooperation in Form einer örtlichen oder überörtlichen Berufsausübungsgemeinschaft (BAG) bietet eine ganze Reihe von Vorteilen: Durch eine bestmögliche Ausnutzung von Ressourcen lassen sich die Kosten senken. Und auch die Chancen der beruflichen Selbstverwirklichung können in einer Kooperation höher sein als in einer Einzelpraxis. Vorteile gibt es außerdem für den Fall einer Praxisabgabe beziehungsweise der Entsprechung dazu bei einem BAG-Gesellschafter, das heißt der Abgabe seines Gesellschaftsanteils.

Durch die frühzeitige Aufnahme des Übernehmers als Jobsharing-Gesellschafter hat der Senior - vielleicht erstmals in seinem langen Berufsleben - die
Möglichkeit kürzerzutreten, indem er seine Arbeitszeit mit dem späteren Übernehmer teilt. Ein Timesharing oder reduzierte Arbeitszeiten für den Senior müssen jedoch im Gesellschaftsvertrag geregelt werden, empfiehlt der Münchner Fachanwalt für Medizinrecht Achim Röschmann bei einer Seminarveranstaltung der Deutschen Apotheker- und Ärztebank in Rosenheim.

Im Falle einer Übergangsgemeinschaft durch Jobsharing muss außerdem beachtet werden, dass diese nur um den Preis von Leistungs- und damit Abrechnungsbeschränkungen der gesamten BAG möglich ist. Ist dieser Preis zu hoch, sollte der Senior nach Angaben des Münchner Medizinrechtlers darüber nachden- ken, die Hälfte seiner Zulassung freizugeben. Bei einem Teilversorgungsauftrag nach $\$ 19$ a Abs. 2 der Zulassungsverordnung muss der Arzt nur für zehn Sprechstunden pro Woche zur Verfügung stehen. Bei diesem Modell der Zulassungsteilung ist jedoch Vorsicht geboten: Kommt es vor der Übergabe zum Streit, ist nicht immer sicher, ob der Übernehmer die halbe Zulassung wieder zurückgibt. Dieses Risiko lässt sich umgehen, indem der Übernehmer während der Übergabezeit lediglich als Angestellter auf der Grundlage eines Verzichts auf eine halbe Zulassung tätig wird und der Senior dennoch seine Arbeitszeit reduziert. Legt aber der Übernehmer, wie häufig, großen Wert darauf, schon in der Übergangszeit Gesellschafter zu sein, muss das Rückgaberisiko durch einen guten Vertrag mit Vertragsstrafenregelung minimiert werden.

Jürgen Stoschek 